\title{
Experience of Obtaining the Plan of Experiments, with Using Models of Interaction of Charged Particles
}

\author{
Normunds Kante, Juris Lavedels, Nikolajs Kriščuks
}

\begin{abstract}
In this article a method of obtaining an experiment plan in a fragment of multidimensional space is analyzed and improved. The method is based on an assumption that particles will distribute evenly in an infinite space with constant charged particle density. To obtain the experiment plan, the infinite multidimensional space is replaced with a hypercube whose surface models influence of the surrounding infinite space. Software is developed and practical results in obtaining experiment plan in two-dimensional space are acquired. Two-dimensional space allows developing of a methodology and algorithm for obtaining experiment plan while providing a simple visualization of the solution. Acquired results in two-dimensional space give an opportunity to create methods for obtaining experiment plan in a hypercube of multidimensional space.
\end{abstract}

Keywords: Experiment plan, charged particle interaction, Latin cube, Hypercube, Uniform distribution.

\section{INTRODUCTION}

Computerized mathematical modeling and metamodeling constitutes an increasingly important role in the development of technical objects and systems. An indispensable part of computerized modeling is the experiment plan [1].

The classic experiment planning method anticipates all variables, except one that is changed during the experiment, to be recorded beforehand, which is the basis of univariate experiments. To use univariate experiment for general research in multivariate processes, a large amount of experiments must be performed. This type of approach is not advisable while researching multivariate systems due to the amount of experiments necessary. To perform multivariate experiments it is necessary to use static experiment planning methods. Algorithms and different mathematical methods are necessary, while formalizing research action and choosing the strategy that gives an opportunity to make important decisions after each series of experiments, to lower the amount of possible experiments.

Experiment plan obtaining is often based on Latin hypercube methods [2] and [3]. Latin hypercube is usually used to acquire experiment plan for an experiment with two to four independent variables. As a result of the rapid development of technology it has become necessary to obtain experiment plan for experiments with eight, ten or even more independent variables. With such a large number of variables Latin hypercube method loses its efficiency. Consequently, it is necessary to research and develop other approaches for obtaining experiment plan [4].
In the end of last century a scientist from Riga Polytechnic Institute - V. Eglājs - suggested that charged particle coordinates in equilibrium state could be used as an experiment plan (in finite space) [5].

\section{MAIN ASSUMPTIONS}

Article [5] describes how an experiment plan can be obtained by modeling particle interaction in a hypercube whose surface models influence of the surrounding multidimensional infinite space. Figure 1 shows research schema for two-dimensional (square) case.

Replacing the infinite space with a square whose surface imitates the influence of the surrounding space causes a methodic mistake, which needs to be minimized. In this article two methods for replacing the infinite space are analyzed [5]:

- Wallpaper - part of the space, which infinitely repeats itself (Fig. 2. a).

- Mirror - part of the space, which does not allow charged particles to leave the space by creating mirror images of the particle (Fig. 2. b). 

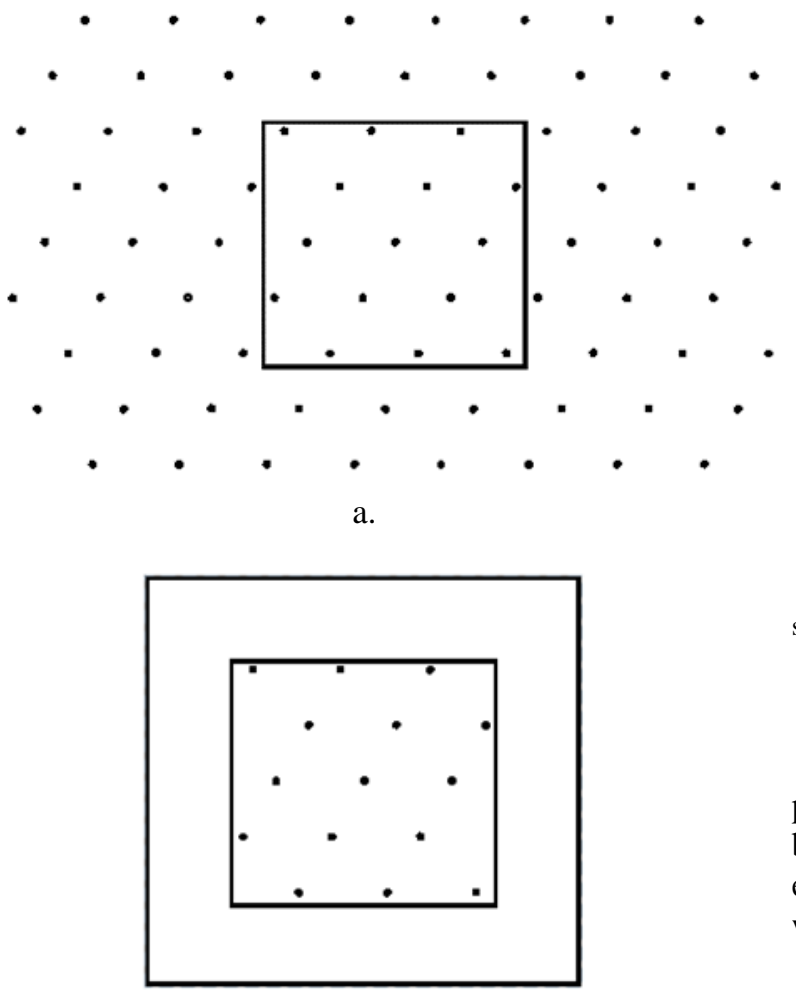

b.

Fig 1. Research schema [6]. a. Square as a fragment of an infinite two dimensional space. b. Square and its perimeter area that imitates the influence of the surrounding infinite space.

\section{CALCULATION SCHEMA}

A calculation area is chosen which contains a certain number of electrons. Around the perimeter of the chosen area identical areas with matching particle (phantom) positioning are placed. One or more phantom rings can be placed around the calculation area. Phantom particles move analogous to the corresponding particle in the calculation area.

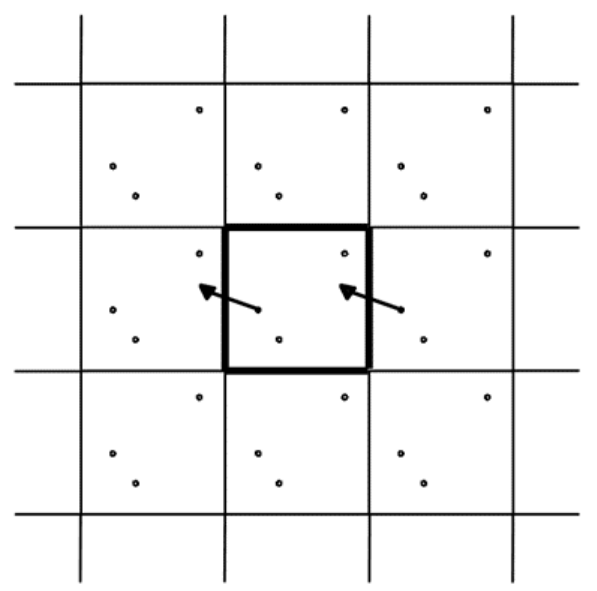

a.

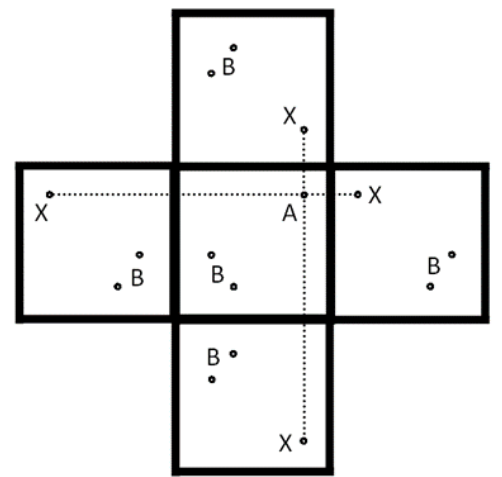

b.

Fig. 2. Replacing of an infinite two-dimensional space with a square and an imitator of the effects of the surrounding space [7]. a.

Replacement method "Wallpapers", b. Replacement method "Mirror"

Figure 2 shows that if as a result of calculation a particle is pushed out of the calculation area it becomes a phantom, while another particle (phantom) enters the calculation area replacing the particle that was pushed out.

\section{SINGLE CHARGED PARTICLE}

DISPLACEMENT CALCULATION SCHEMA

Calculations are made for each particle by determining the particles coordinates at its equilibrium state as shown in Figure 3.

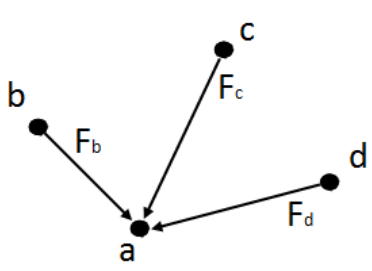

a.

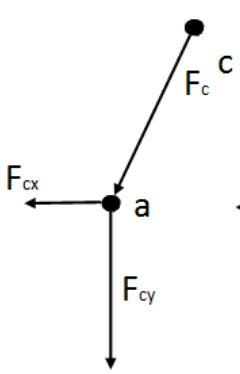

C.

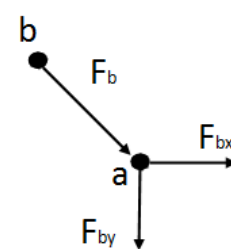

b.
Fig. 3. Finding the equilibrium state of a charged particle [8]

To determine the new coordinates for the particle $a$, we need to find and calculate the following:

- All forces that affect the particle - $F_{b}, F_{c}, F_{d}$. (2. att. a)

$$
F_{a i}=C \frac{1}{r_{a i}^{2}}, \text { where }
$$


- $a$ - charged particle;

- $C$ - coefficient;

- $r_{a i}$-distance between particle $a$ and particle $i$ $(b, c, d)$;

- Forces are divided into components $x$ and $y-$ $F_{b x}, F_{b y}, F_{c x}, F_{c y}, F_{d x}, F_{d y}$. (Fig. 2. b, c, d);

- Cumulative force $F_{x}$ is calculated for the particle:

$$
F_{x}=F_{b x}+F_{c x}+F_{d x}
$$

- Cumulative force $F_{y}$ is calculated for the particle:

$$
F_{y}=F_{b y}+F_{c y}+F_{d y}
$$

- Absolute cumulative force that is affecting the particle:

$$
F=\sqrt{F_{x}^{2}+F_{y}^{2}}
$$

- Particle is moved a certain distance $l$, for example, half of the distance to its nearest neighbor;

- The new coordinates of the particle are determined by dividing displacement of the particle into its vector components.

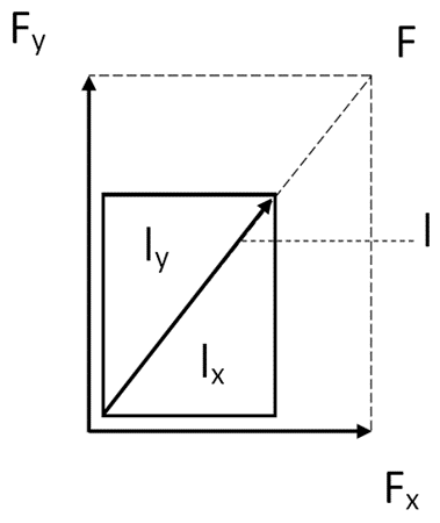

Fig. 4. Determining displacement $l_{x}$ and $l_{y}$

$$
\begin{aligned}
& l_{x}=\frac{F_{x}}{F} * l \\
& l_{y}=\frac{F_{y}}{F} * l, \text { where }
\end{aligned}
$$

- $\quad l_{x}$ - displacement on $x$ axis;

- $l_{y}$ - displacement on $y$ axis;

- $F$ - cumulative force;

- $F_{x}$ - force on $x$ axis;

- $F_{y}$ - force on $y$ axis;

- $l$-displacement of the particle;

- Each particle is moved until the displacement no longer lessens the force that is affecting the particle, or energy level of the entire system no longer decreases. Then the positioning of the charged particle is optimized.

The proposed method also makes it possible to obtain experiment plans containing concentration or distribution in specific areas of the calculation area. Using this special approach, the process can be improved if the optimal particle distribution area for obtaining experiment plan is known. This can be easily achieved by assigning charged particle coordinates in calculation as a function.

$$
F=\frac{q_{1}(x, y) * q_{2}(x, y)}{r^{2}}
$$

By assigning the value of the charge as a function $\mathrm{q}=\mathrm{q}(\mathrm{x}, \mathrm{y})$ of coordinates $\mathrm{x}$ and $\mathrm{y}$, it is possible to obtain experiment plan with a concentration in areas where the value of $\mathrm{q}(\mathrm{x}, \mathrm{y})$ is low or with a distribution in areas where the value of $q(x, y)$ is higher.

\section{ITERATIVE PROCESS OF CHARGED PARTICLE DISPLACEMENT}

To acquire experiment plan or normal distribution of charged particles in multidimensional hypercube calculations are made that are analogous to a theoretical experiment. At first, all charged particles are freely distributed. Coordinates of these particles can be generated using random number generator or using different types of functions as input parameters. Phantom areas that imitate the surrounding infinite space are placed around the perimeter of the calculation area. Whenever a particle in the calculation area is moved, the corresponding particle in the phantom areas is also moved. When calculating the displacement of a particle all phantoms of that particle are taken into account.

The following process executed for all charged particles:

1. Position of all particles is recorded;

2. Particle whose displacement will be calculated is determined;

3. New coordinates, where cumulative force affecting this particle is zero or as low as possible, are calculated for the chosen particle taking into account other particle effect and all phantom particle effect in all phantom areas on the chosen particle;

4. Phantom particles of the chosen particle are adjusted based on new coordinates;

5. It is determined whether the placement of the particle at its equilibrium state improves the quality of the entire system - will this reduce the absolute amount of unbalanced forces in the system, equalizing the distance between particles;

6. Return to step 1.

This process is used to iteratively optimize positioning of all particles and the state of the entire system. Executing a final number of iterations a particle positioning is obtained that is close to normal distribution. Quality indicators of the particle normal distribution are:

- Cumulative force that affects the particle and that cannot be minimized;

- Distance from the chosen particle to the nearest charged particle;

- Vectorial sum of the interaction forces in the system; 
- Sum of the interaction forces on each of the axis;

Practical implementation of methodology provides both optimization of charged particle coordinates in a single iteration and iterations for single particle state optimization. The latter gives an opportunity to, for example, select particles that have a distance to the nearest particle that is larger than the average distance and optimize coordinates for these particles several times, thus increasing convergence of the solution.

Numerical calculations show that in separate cases the system reaches a state where optimization process stops. In these cases the convergence of the process can be restored by freely changing the coordinates of some particles (creating a mutation as in genetic algorithm).

Proposed methodology is practically realized by creating software, many experimental calculations are made and practical results are obtained.

\section{PRACTICAL RESULTS AND CONCLUSION \\ Described methodology was practically} implemented and software was created. Calculations were made to obtain experiment plan in twodimensional space (see results in Figure 5 and Figure $6)$.

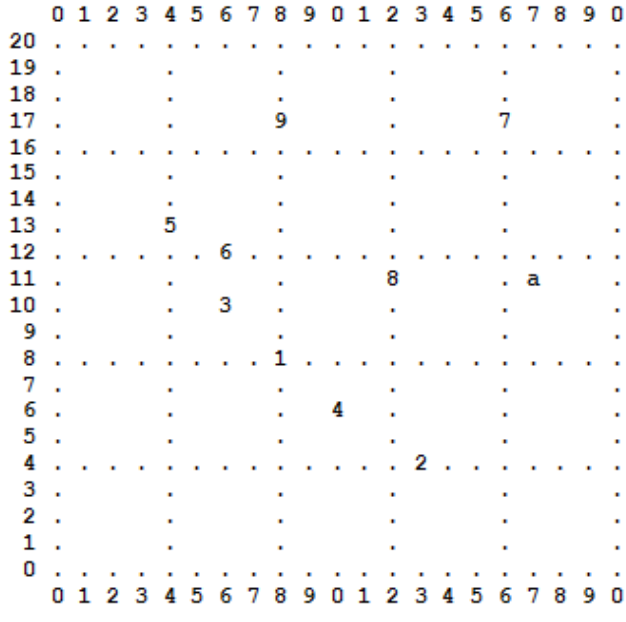

$$
\begin{array}{rcrcc}
i & f & \multicolumn{1}{c}{\mathrm{fx}} & \mathrm{fy} & \mathrm{Rmin} \\
1 & 3.00 \mathrm{E}+0001 & -6.06 \mathrm{E}+0000 & -2.94 \mathrm{E}+0001 & 0.14 \\
2 & 2.87 \mathrm{E}+0001 & 2.46 \mathrm{E}+0001 & -1.48 \mathrm{E}+0001 & 0.22 \\
3 & 8.99 \mathrm{E}+0001 & -4.37 \mathrm{E}+0001 & -7.86 \mathrm{E}+0001 & 0.10 \\
4 & 5.64 \mathrm{E}+0001 & 2.42 \mathrm{E}+0001 & -5.09 \mathrm{E}+0001 & 0.14 \\
5 & 7.56 \mathrm{E}+0001 & -5.24 \mathrm{E}+0001 & 5.44 \mathrm{E}+0001 & 0.14 \\
6 & 8.18 \mathrm{E}+0001 & 1.23 \mathrm{E}+0001 & 8.08 \mathrm{E}+0001 & 0.10 \\
7 & 1.04 \mathrm{E}+0001 & 8.90 \mathrm{E}+0000 & 5.30 \mathrm{E}+0000 & 0.32 \\
8 & 3.16 \mathrm{E}+0001 & 2.67 \mathrm{E}+0001 & 1.69 \mathrm{E}+0001 & 0.25 \\
9 & 1.96 \mathrm{E}+0001 & 1.28 \mathrm{E}+0000 & 1.95 \mathrm{E}+0001 & 0.28 \\
10 & 2.56 \mathrm{E}+0001 & -3.24 \mathrm{E}+0001 & 2.43 \mathrm{E}+0001 & 0.15
\end{array}
$$$$
\text { Sum } 5.28 \mathrm{E}+0000 \quad 4.21 \mathrm{E}+0000-3.19 \mathrm{E}+0000 \quad 0.30
$$

Fig. 5. Starting position of 10 points in calculation area.

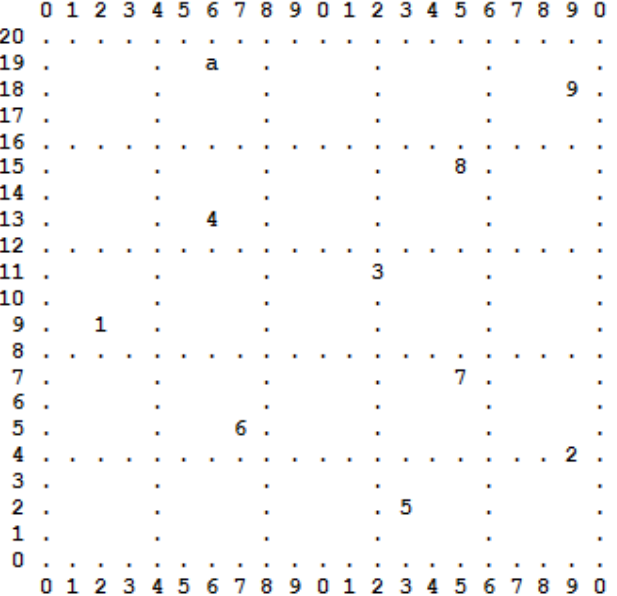

$\begin{array}{rrrrr}i & f & f x & f y & \text { Rmin } \\ 1 & 6.41 \mathrm{E}+0000 & -3.49 \mathrm{E}+0000 & 5.38 \mathrm{E}+0000 & 0.28 \\ 2 & 1.01 \mathrm{E}+0001 & 8.54 \mathrm{E}+0000 & -5.43 \mathrm{E}+0000 & 0.26 \\ 3 & 2.23 \mathrm{E}+0000 & -1.54 \mathrm{E}+0000 & -1.62 \mathrm{E}+0000 & 0.24 \\ 4 & 1.43 \mathrm{E}+0000 & -1.43 \mathrm{E}+0000 & -6.43 \mathrm{E}-0002 & 0.28 \\ 5 & 3.93 \mathrm{E}+0000 & -3.07 \mathrm{E}+0000 & 2.46 \mathrm{E}+0000 & 0.25 \\ 6 & 2.34 \mathrm{E}+0000 & -1.10 \mathrm{E}+0000 & 2.07 \mathrm{E}+0000 & 0.31 \\ 7 & 9.66 \mathrm{E}-0001 & -6.25 \mathrm{E}-0001 & 7.36 \mathrm{E}-0001 & 0.24 \\ 8 & 1.92 \mathrm{E}+0000 & -1.90 \mathrm{E}+0000 & 2.88 \mathrm{E}-0001 & 0.24 \\ 9 & 1.03 \mathrm{E}+0001 & 8.60 \mathrm{E}+0000 & -5.64 \mathrm{E}+0000 & 0.30 \\ 10 & 4.39 \mathrm{E}+0000 & -3.99 \mathrm{E}+0000 & 1.82 \mathrm{E}+0000 & 0.30 \\ \text { Sum } & 4.40 \mathrm{E}+0001 & 3.64 \mathrm{E}-0011 & 1.36 \mathrm{E}-0010 & 2.72\end{array}$

Fig. 6. Position of 10 points after 25 iterations in calculation area.

$\begin{array}{lllllllllllllllllllll}0 & 1 & 2 & 3 & 4 & 5 & 6 & 7 & 8 & 9 & 0 & 1 & 2 & 3 & 4 & 5 & 6 & 7 & 8 & 9 & 0\end{array}$

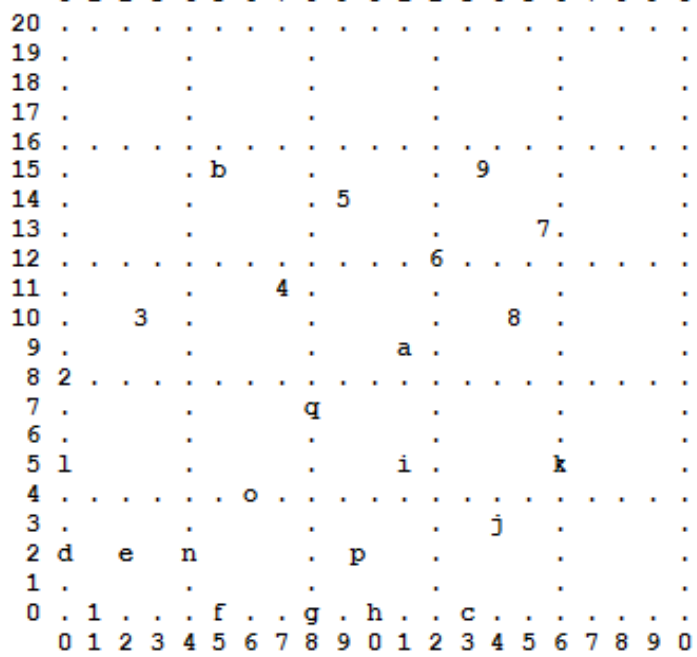

Fig. 7. Example of an experiment plan with a particle concentration around the coordinates $(0,0)$

It must be noted that the quality and sped of the replacement method „Wallpapers" is dependent on charged particle (experiment) amount - the amount of charged particles in the calculation area might be detrimental. It was empirically detected that, if result cannot be found due to poor convergence of the method, it is necessary to increase the amount of phantom rings around the calculation area to obtain a qualitative experiment plan. I was also empirically detected that by changing the parameters, the location of the particle in the calculation area can be adjusted as needed. Even though several qualitative results are 
Environment. Technology. Resources, Rezekne, Latvia Proceedings of the $11^{\text {th }}$ International Scientific and Practical Conference. Volume II, 47-51

obtained using calculation schema, methodology still needs to be optimized.

Following conclusions can be made:

- Experiment plan obtaining method based on charged displacement modeling was practically implemented;

- The developed method is implemented in the Java programming language and it can be used just like other methods [1];

- Developed method was tested for experiments in two-dimensional space. It can be easily used for a larger number of dimensions;

- Method can also be used to obtain an experiment plan for unevenly distributed particles. In this case the charge of the particle must be defined as function of coordinates;

- The results acquired are interesting and the work on the methodology should be continue.

\section{REFERENCE}

[1] J. Auzins, A. Janusevskis. „Experiment planning and analysis" Rīga, RTU 2007, ISBN 97-9984-32-157-8

[2] N. A. Butler. „Optimal and Orthogonal Latin Hypercube Designs for Computer Experiements". Biometrika 2001., 88, 847-857

[3] R. J. Iman and M. J. Shortencarier., A FORTRAN77 „Program and User's Guide for Generation of Latin
Hypercube and Random Samples for Use with Computer Models", Sandia National Laboratories, Albuquerque, NM 1984., NUREG/CR-3624, SAND83-2365

[4] P. Audze and V. Eglājs. „New approach to the design of multifactor experiments" Problems of Dynamics and Strengths 35, Zinatne Publishing House, Riga 1977, 104-107

[5] N. Kante, J. Lavendels, "Obtaining The Plan Of Experiments, On The Basis Of Modeling Of Interaction Of Charged Particles" - International Conference on "Theory and practice of simulation modeling and creation of simulators: the accumulation and processing of information, information models, information tools" (in Russian), Penza, Penza State Technological University, 2015, pp.24-28. ISBN 97859907043-2-9., DOI 10.18411/2016-031-001

[6] N. Kante, M. Kryshchuk, J. Lavendels., "Experiment Plan as a Discreet System Equilibrium State." Applied Computer Systems. Vol.20, 2016, pp.49-52. ISSN 2255-8683. e-ISSN 2255-8691. Available from: doi:10.1515/acss-2016-0015

[7] N. Kante, J. Lavendels. „Equilibrium state simulation of charged particles based experiment plan obtaining method in discrete systems" Contemporary Information Technologies, Penza State University, 2016, ISSN 1815-2724

[8] N. Kante, M. Kryshchuk, J. Lavendels, "Charged Particle Location Modeling Based Experiment Plan Acquisition Method." Procedia Computer Science, 2017, Vol.104, pp.592-597. ISSN doi:10.1016/j.procs.2017.01.177 